\title{
GEOBIA E MINERAÇÃO DE DADOS PARA CLASSIFICAÇÃO DE IMAGENS DE ALTÍSSIMA RESOLUÇÃO ESPACIAL
}

\author{
Adalto Moreira Braz ${ }^{1}$ \\ Thales Sehn Körting ${ }^{2}$ \\ Alécio Perini Martins ${ }^{3}$ \\ Amanda Moreira Braz ${ }^{4}$
}

Resumo: Este trabalho apresenta os resultados de classificação (GEOBIA e mineração de dados) para a cobertura da terra em imagens de altíssima resolução espacial, além de apresentar uma discussão sobre a viabilidade de classificações supervisionadas em imagens obtidas por VANT. A área teste inclui duas quadras e uma área verde na cidade de Jataí (GO), imageadas com um Drone DJI Phantom 4 Advanced em 29/10/2018. O processamento foi realizado a partir do SIG TerraView 5.3.3 plugin GeoDMA 2.0, com resultados comparados a classificadores disponibilizados pelo SIG ArcGIS 10.6.1®. A mineração de dados foi a técnica mais adequada para a classificação, apresentando uma matriz com taxa de acerto superior a $82 \%$, concordância excelente apesar dos erros identificados. No processo de classificação supervisionada, foi obtido um acerto de $69 \%$ e, na classificação não supervisionada, o acerto foi de $44 \%$. Os resultados da GEOBIA/mineração de dados foram considerados satisfatórios (Kappa igual a 82\%), quando comparados a outros classificadores (regão e pixel a pixel), mas ainda exigindo atividades de pósclassificação para correção das confusões apresentadas.

Palavras-Chave: VANT; Sensoriamento Remoto; ARP; Drone; Cobertura da Terra;

\section{GEOBIA AND DATA MINING FOR HIGH SPATIAL RESOLUTION IMAGE CLASSIFICATION}

\begin{abstract}
In this paper we present the classification results (GEOBIA and data mining) for land cover in high spatial resolution images, besides presenting a discussion about the viability of supervised classifications in UAV images. The study area includes two blocks and a green area in the city of Jataí, Goiás state, Brazil. The images were obtained with a DJI Phantom 4 Advanced Drone in 2019/10/29. Processing was performed using the GIS TerraView 5.3.3 plugin GeoDMA 2.0, and the results were compared to well known classification algorithms provided by ArcGIS 10.6.1®. Data mining was the most appropriate technique for classification, presenting a matrix with an overall accuracy (OA) higher than $82 \%$, which we consider as an excellent agreement despite minor errors. In the supervised classification process, we obtained

\footnotetext{
${ }^{1}$ Doutor em Geografia pela Universidade Federal de Goiás - UFG. Email: adaltobraz.geografia@gmail.com

2 Professor dos Programas de Pós-Graduação em Sensoriamento Remoto e Computação Aplicada (INPE). Email: thales.korting@inpe.br

${ }^{3}$ Professor Adjunto da Universidade Federal de Goiás, Campus - Jataí (GO). Email: alecioperini@ufg.br

4 Geógrafa formada pela Universidade Federal do Mato Grosso do Sul, Campus Três Lagoas (MS). Email: amandabraz.geo@gmail.com
} 
$69 \%$ OA (substantial agreement) and 44\% OA (moderate agreement) in the unsupervised classification. The results of GEOBIA/data mining were considered satisfactory (Kappa is equal to $82 \%$ ) when compared to other classifiers (region and pixel by pixel), but still requiring post-classification activities to correct the confusion presented.

Keywords: UAV; Remote Sensing; RPA; Drone; Land Cover;

\section{INTRODUÇÃO}

Nos últimos anos a utilização de Veículos Aéreos Não Tripulados (VANT) ${ }^{5}$ se consolidou no Brasil e no mundo. Notórios pela agilidade na tomada de imagens, facilidade no processamento e bom desempenho proporcionado principalmente pelas resoluções espaciais (centimétricas).

Comparado a outros sensores disponíveis no mercado atual, os VANTs se destacam devido à sua resolução espacial, liberdade ao operador do equipamento para a obtenção de imagens em diferentes momentos, facilidade no desenvolvimento de planos de voos, além da grande possibilidade de se estruturar uma base com imagens multitemporais. Permitindo agilidade, qualidade e baixo custo aos usuários (BRAZ et al., 2015, p. 2751).

Com o advento dos VANTs o espectro de possibilidades para a mensuração e cadastro urbano, sobretudo no que tange ao menor custo para aquisição de imagens, à alta repetibilidade na tomada das imagens e a precisão para grandes escalas de mapeamento. A aplicação de VANTs é recomendada para o mapeamento urbano pois, proporciona a extração de informações com altíssima resolução espacial (nível de detalhamento), permitindo a representação cartográfica de áreas urbanas, comumente marcadas pela alta complexidade pelo elevado número de elementos (classes) a serem cartografadas.

Os ambientes urbanos são muito complexos porque misturam diversas características de elementos que podem gerar uma relação confusa entre o objeto e seu espectro, como por exemplo, objetos diferentes podem ter o mesmo espectro ou os mesmos objetos podem ter o espectro diferente (CHEN et al., 2009; MARTINS, 2012).

A rápida evolução tecnológica do sensoriamento remoto vem possibilitando a obtenção de imagens de altíssima resolução espacial e temporal, com isso, sua utilização em ambientes urbanos tem sido ampliada e favorecida, em tomadas de imagens para grande escala, tão necessárias ao planejamento urbano (Quadro 1), que tem requerido intensos mapeamentos para atualização da informação geográfica (CERQUEIRA e ALVES, 2010).

Conforme a classificação de Ehlers, Janowsky e Gaehler (2002) as imagens de altíssima resolução espacial (ultra high spatial resolution) apresentam pixels menores que $\leq 1,0 \mathrm{~m}$. Cabe lembrar que a resolução espacial de imagens aéreas é tratada pelo termo distância de amostragem do solo (Ground Sample Distance - GSD). O GSD é, portanto, a distância entre os pontos centrais de cada amostragem do solo,

\footnotetext{
${ }^{5}$ Entende-se aqui, por VANT, o nome que mais se popularizou para objeto remotamente pilotados (não tripulados). Estes, ainda, podem ser conhecidos como Drones, Aeronave Remotamente Pilotada (ARP), Remotely-Piloted Aircraft (RPA), Unmanned Aerial Vehicle (UAV) entre outros.
} 
representado pelos pixels das imagens. O que significa que o GSD indica o tamanho de cada pixel da imagem e, consequentemente, sua resolução espacial. Isso indica que quanto menor for o valor do GSD, melhor e maior será a resolução espacial da imagem. Por isso, optou-se por utilizar o termo mais comum, de resolução espacial.

Quadro 1. Resoluções espaciais em imagens para aplicações urbanas

\begin{tabular}{|c|c|c|c|}
\hline \multicolumn{2}{|c|}{ Escalas de aplicação em áreas urbanas } & \multicolumn{2}{|c|}{ Imagens a serem utilizadas } \\
\hline Aplicações & Escalas & Imagens & Resolução \\
\hline $\begin{array}{c}\text { Gestão Técnica } \\
\text { Mapeamento } \\
\text { Básico }\end{array}$ & $1: 200$ para 1:500 & Ortofoto & $>20 \mathrm{~cm}$ \\
\hline $\begin{array}{c}\text { Planejamento } \\
\text { urbano }\end{array}$ & $1: 5000$ para 1:10000 & Ortofoto & $\begin{array}{c}50 \mathrm{~cm} \text { para } 1 \\
\mathrm{~m}\end{array}$ \\
\hline Prospecção & $\begin{array}{c}1: 25000 \text { para } \\
1: 1.000 .000\end{array}$ & $\begin{array}{c}\text { Imagem de } \\
\text { Satélite }\end{array}$ & $>10 \mathrm{~m}$ \\
\hline
\end{tabular}

Fonte: Adaptado de Puissant e Weber (2002).

Novas plataformas para aquisição de imagens exigem também novas abordagens para 0 tratamento de imagens e da informação geográfica. Neste contexto, a geoinformação apresenta o estudo das questões fundamentais da informação geográfica, motivada pela necessidade de melhorar as (geo)tecnologias que tratam, sobretudo, das informações espaciais (GOODCHILD, 2011).

Em se tratando de classificação de imagens, a abordagem pixel-a-pixel vem tendo seus resultados questionados, sobretudo pelo chamado efeito "sal e pimenta" (PILZ e STROBL, 2007). Nos VANTs, uma das características mais importantes é a da resolução espacial. Em vista disso, as imagens passam a representar quantidades ainda mais elevadas de pixels (linhas e colunas) e, consequentemente, mais características texturais.

A resolução espacial centimétrica é um diferencial para mapeamentos em grandes escalas, ao passo que a alta variabilidade radiométrica se torna uma barreira para classificação de suas imagens (LECHNER et al., 2012; RUIZ, GUASSELLI e HACKMANN, 2016). Sobretudo no contexto das áreas urbanas, onde em pequenas áreas, há uma quantidade razoável de diferentes estruturas e elementos, que refletem numa grande quantidade de classes de uso da terra representada pela imagem.

A primeira e mais difundida solução para amenizar este problema é a segmentação de imagens, procedimento que antecede a classificação. Neste processo, são agrupados os pixels com atributos espectrais semelhantes. Todavia, a constante evolução dos produtos gerados por sensoriamento remoto (tanto satélites, quanto VANTs) tem exigido, além dos atributos espectrais, a consideração por atributos espaciais (geométricos), escalares e contextuais. Essa técnica ficou conhecida como Análise de Imagens Baseada em Objetos Geográficos (GEOBIA, na sigla em inglês) ${ }^{6}$.

A respeito da classificação de imagens em sensoriamento remoto, Blaschke (2010) chamou atenção para a GEOBIA, que tem obtido progressos consideráveis no que tange à extração de informações espaciais e espectrais de imagens.

De acordo com Hay e Castilla (2008) a GEOBIA é:

um ramo de Ciência da Informação Geográfica (GIScience ou Geoinformação) dedicada ao desenvolvimento de métodos

\footnotetext{
${ }^{6}$ Geographic Object-Based Image Analysis (GEOBIA)
} 
automatizados para particionar imagens de sensoriamento remoto em objetos espaciais significativos, e avaliar suas características através de escalas espaciais, espectrais, temporais e gerar novas informações geográficas (HAY e CASTILLA, 2008, p. 77).

Com relação à complexidade de se obter resultados satisfatórios na classificação de imagens de VANT, além da GEOBIA, existe a mineração de dados. Esta técnica, além de se apropriar dos objetos oriundos da segmentação pela GEOBIA, bem como de sua extração dos atributos espaciais, espectrais e contextuais, emprega mecanismos estatísticos para gerar um treinamento supervisionado com o objetivo de prever melhores resultados para a classificação da imagem.

Haja vista que a capacidade humana de análise e compreensão de grandes conjuntos de dados é inferior à capacidade de coleta e armazenamento desses dados, Fayyad, Shapiro e Smyth (1996b) apresentaram a mineração de dados como uma nova geração de técnicas computacionais necessária para a extração de conhecimentos úteis de quantidades cada vez maiores de dados produzidos.

A mineração de dados pode ser entendida como um processo de identificação de (novos) padrões que, dependendo da área de aplicação, sejam válidos, úteis e compreensíveis. Ainda, a mineração de dados abrange procedimentos de descoberta de conhecimentos (inéditos) através da análise de dados por meio de algoritmos para, finalmente, produzirem um conjunto de padrões sobre os dados (FAYYAD, SHAPIRO e SMYTH, 1996a).

A mineração de dados é uma etapa para extrair padrões (modelos) de dados [...] como preparação de dados, seleção de dados, limpeza de dados, incorporação de conhecimento prévio apropriado e interpretação adequada dos resultados da mineração, que garantem que o conhecimento útil seja derivado dos dados (FAYYAD, SHAPIRO e SMYTH, 1996b, p. 29).

Devido à alta precisão centimétrica das imagens de VANT, quando estas imagens são segmentadas, são gerados inúmeros objetos geográficos. No contexto da GEOBIA, pode-se ainda extrair muitos atributos para cada um dos objetos geográficos. Sendo assim, é possível optar pela mineração de dados para extrair conhecimentos úteis das imagens, neste caso, almejando uma classificação de cobertura da terra precisa e mais próxima possível da realidade.

Com relação à aplicação de técnicas de mineração de dados para classificação de imagens de VANT, é possível citar Ruiz, Guasselli e Hackmann (2016), que utilizaram segmentação através do Sistema de Processamento de Informações Georreferenciadas (SPRING) e aplicação de análise de imagens baseada em objetos (Object-based Image analysis - OBIA) através de linguagem de programação Python, gerando as classificações (métodos de Máquina Vetores de Suporte, K-Vizinhos mais Próximos e Floresta Aleatória) com a biblioteca de mineração de dados Scikit-Learn e validações estatísticas com a biblioteca Stats Model.

Gerke (2017) utilizou mineração de dados para estimar a produtividade da cultura de trigo, através dos valores de reflectância extraídos das imagens e minerados por meio do software Weka, utilizando o algoritmo SMORreg, baseado em máquina de vetor de suporte para regressão. Reafirma-se, então, que os trabalhos além de recentes são ainda escassos na bibliografia especializada.

Por conseguinte, o objetivo deste trabalho é comparar e avaliar os resultados de classificação para cobertura da terra em imagens de altíssima resolução, aplicando três métodos de classificação, a GEOBIA (mineração de dados), iso cluster (não 
supervisionada) e maximum likelihood (supervisionada). Além disso, o trabalho pretende apresentar uma discussão sobre a viabilidade de classificações supervisionadas em imagens de VANTs.

A área escolhida para aplicação da GEOBIA e mineração de dados para classificação da imagem aérea foi uma parte (área-teste) do bairro Filostro Machado na cidade de Jataí, Estado de Goiás, Brasil.

\section{PROCEDIMENTOS}

A área-teste para classificação é um recorte de duas quadras e uma área verde no setor Filostro Machado na cidade de Jataí (GO) com 19.090,16m², escolhida pela densidade de construções e diferenças de telhados. O setor localiza-se na zona norte da cidade, em área periférica ocupada principalmente por população de classe médiabaixa. Ao todo são 05 quadras e 114 lotes com uma média de $250 \mathrm{~m}^{2}$ e uma área verde central com quadra de esportes, bancos, espaço gramado e arborização esparsa.

Os procedimentos adotados obedecem à organização do fluxograma abaixo (Figura 1) são detalhados nos tópicos a seguir:

Figura 1. Fluxograma de procedimentos para classificação da imagem de VANT

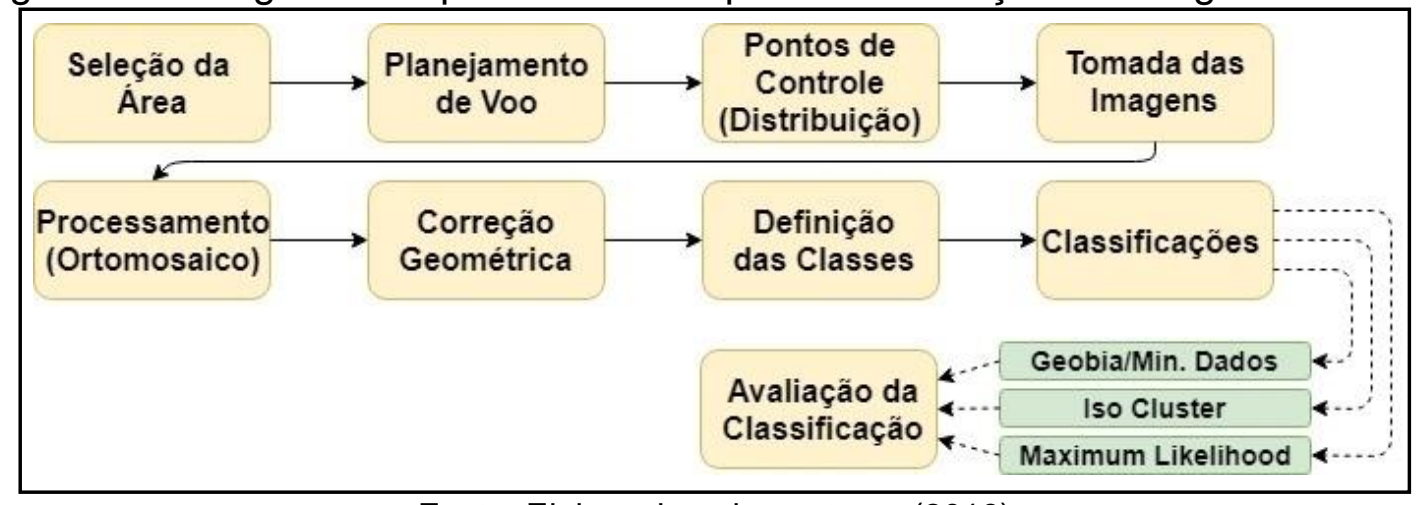

Fonte: Elaborado pelos autores (2019)

\section{AQUISIÇÃO DAS IMAGENS E PROCESSAMENTO DO ORTOMOSAICO}

A classificação por GEOBIA e mineração de dados foi elaborada a partir de imagens do VANT DJI Phantom 4 Advanced, tomadas na data 29/10/2018, próximo às $11 \mathrm{~h}: 30 \mathrm{~min}$. Para a elaboração do plano de voo utilizou-se o aplicativo gratuito para sistema Android DroneDeploy, com altura de 100 metros em relação ao solo, velocidade média de $12 \mathrm{~m} / \mathrm{s}$ e overlap de $75 \%$ (frontal e lateral). As fotografias foram ajustadas e mosaicadas no Agisoft PhotoScan (versão de teste) e exportadas em formato TIFF com 2,6cm de resolução espacial e compostas em RGB cor verdadeira.

Em campo foram estabelecidos 10 pontos de controle, marcados no asfalto com tinta branca em um espaço de $40 \mathrm{~cm} \times 40 \mathrm{~cm}$. As coordenadas foram obtidas com uso do Global Navigations Satellite System (GNSS) Trimble Geoexplorer 2008 series e passaram por correção diferencial no software Pathfinder Office $\AA$ utilizando a base da Rede Brasileira de Monitoramento Contínuo dos Sistemas GBSS (RBMC - Estação GOJA). Após o processamento obteve-se coordenadas com um erro médio de $10 \mathrm{~cm}$, utilizadas para georreferenciar o ortomosaico Sistema de Informações Geográficas (SIG) no ArcGIS 10.6.1 licenciado para o Laboratório de Geoinformação da UFG/Regional Jataí. 


\section{CLASSIFICAÇÃO DAS IMAGENS}

Para a classificação (GEOBIA e mineração de dados), utilizou-se o SIG TerraView 5.3.3 e do plugin GeoDMA 2.0 (ambos open source). O TerraView é um SIG capaz de lidar com bancos de dados espaciais, imagens e regiões resultantes do processo de segmentação, além manipular outros dados geográficos, como por exemplo, dados cadastrais.

O GeoDMA é o software responsável pela mineração de dados, a partir de técnicas de aprendizado por máquina, que possibilita a extração de padrões dos objetos obtidos por segmentação. Em termos operacionais, o GeoDMA é capaz de realizar extração e seleção de atributos e organização de árvores de decisão, por mineração de dados, através da exploração de características espaciais, espectrais e de vizinhança (KÖRTING, FONSECA e CÂMARA, 2010; 2013).

A classificação teve início com a equalização do histograma da imagem no TerraView, obtendo valor de 1,7 em ganho e $30 \mathrm{em}$ offset. Em seguida, foram realizados alguns testes em relação aos parâmetros de segmentação da imagem a partir do algoritmo de crescimento de região médio, apontando como melhor resultado o valor 500 de tamanho mínimo dos segmentos, 0,120 para o limiar de similaridade e 7 para sobreposição. $O$ peso de interesse nas bandas de entrada foi determinado em 0,3333 proporcional às três bandas. A segmentação extraiu 21.813 objetos dentro dos $19.090,16 \mathrm{~m}^{2} \mathrm{da}$ área teste (Figura 2).

Figura 2. Exemplo de objetos da segmentação da imagem

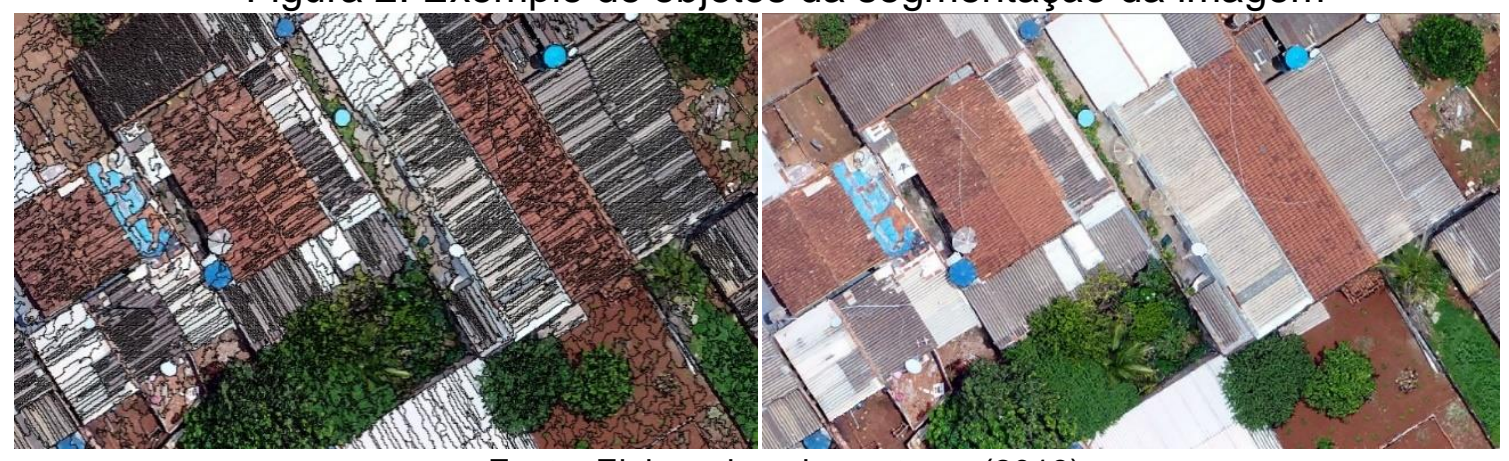

Fonte: Elaborado pelos autores (2019)

Em seguida, iniciou-se a extração dos atributos (espaciais e espectrais) da imagem do VANT. Devido à complexidade dos alvos na imagem, sobretudo devido à quantidade de possíveis classes e a altíssima resolução espacial, optou-se por considerar todos os atributos disponibilizados pelo GeoDMA (Quadro 2). O detalhamento das descrições de cada atributo é disponibilizado por Körting et al. (2013).

Quadro 2. Métricas (espaciais e espectrais) exploradas para a classificação

\begin{tabular}{|l|l|}
\hline \multicolumn{1}{|c|}{ Métricas Espectrais } & \multicolumn{1}{c|}{ Métricas Espaciais } \\
\hline Amplitude & Ângulo do objeto \\
\hline Razão da banda & Área do objeto \\
\hline Soma de pixels no objeto & Área da caixa delimitadora do objeto \\
\hline Contraste de intensidade & Perímetro da caixa delimitadora do objeto \\
\hline Dissimilaridade & Círculo circunscrito ao redor do objeto \\
\hline Energia & Elipse circunscrita ao redor do objeto \\
\hline Entropia & Dimensão Fractal \\
\hline
\end{tabular}




\begin{tabular}{|c|c|}
\hline Homogeneidade & Distância média entre cada vértice do objeto \\
\hline Curtose & Raio do objeto \\
\hline Valor máximo & Altura da caixa delimitadora do objeto \\
\hline Média & Perímetro do objeto \\
\hline Mediana & Relação entre perímetro e área do objeto \\
\hline Valor mínimo & Razão entre área do objeto e raio do objeto \\
\hline $\begin{array}{l}\text { Modo (nível de cinza mais } \\
\text { comum) }\end{array}$ & Largura da caixa delimitadora do objeto \\
\hline Número de bodós do objeto & Compacidade do objeto \\
\hline Skewness dos pixels no objeto & \multirow{4}{*}{$\begin{array}{l}\text { Ajuste do retângulo mínimo fora do objeto e a } \\
\text { proporção entre área do objeto e área do } \\
\text { retângulo }\end{array}$} \\
\hline Desvio padrão & \\
\hline Soma & \\
\hline $\begin{array}{l}\text { Contagem válida de pixels do } \\
\text { objeto }\end{array}$ & \\
\hline Coeficiente de variação & \multirow{2}{*}{$\begin{array}{l}\text { Razão entre perímetro do objeto e raiz } \\
\text { quadrada da área do objeto }\end{array}$} \\
\hline Variância & \\
\hline
\end{tabular}

Fonte: Modificado de Körting et al. (2013); INPE (2018).

A partir disso, definiu-se previamente que a classificação adotaria 9 classes de uso da terra, e deu início à coleta de amostras (amostras), distribuídas em 3.555 amostras: árvore (564 amostras), asfalto (304 amostras), caixa d'água (16 amostras), calçada (372 amostras), grama (500 amostras), telhado cerâmica (789 amostras), telhado fibra (727 amostras), telhado metal (189 amostras), terreno (94 amostras).

Após a coleta, adotou-se o algoritmo C5.0 integrado ao GeoDMA para realizar a classificação, iniciado pela criação da árvore de decisão. O resultado da operação disponibiliza um mapa de padrões com objetos classificados entre as 9 classes escolhidas anteriormente.

Com a finalidade de comparar os resultados do processo de mineração, foram utilizados dois outros classificadores bastante comuns e disponibilizados pela maioria dos Sistemas de Informações Geográficas: a classificação não supervisionada e a classificação supervisionada por segmentação pela obtenção de áreas amostrais. Ambos os procedimentos foram executados no SIG ArcGIS 10.6.1, também utilizado para a geração dos mapas temáticos.

O processo de classificação não supervisionada do ArcGIS 10.6.1 é denominado Iso Cluster e utiliza algoritmos para reconhecer as classes na imagem, sem controle do usuário ou homogeneidade de classes. Os pixels são agrupados (clustering) de acordo com características de área, proximidade e níveis de cinza compondo regiões homogêneas que indicam uma probabilidade de classe.

Para este processamento, foram geradas 100 prováveis classes, com agrupamentos mínimos de 5 pixels e 5 níveis de cinza, que posteriormente foram identificados por interpretação visual e reclassificados para a composição das 09 classes indicadas anteriormente (árvore, asfalto, caixa d'água, calçada, grama, telhado cerâmica, telhado fibra, telhado metal e terreno) pela ferramenta Reclassify.

Ainda no ArcGIS 10.6.1 foi realizada a classificação supervisionada da mesma imagem pelo modelo pixel a pixel (Maximum Likelihood Classification), que demanda um conhecimento prévio do técnico-intérprete da região, sendo que as amostras servem como padrão de comparação para o algoritmo indicar as classes em toda a imagem. $\mathrm{Na}$ etapa de treinamento (aquisição de amostras) foram identificados 10 pontos de cada uma das 09 classes. Este classificador pondera a distância entre os níveis de cinza, utilizando estatísticas do treinamento para calcular a probabilidade de 
um pixel pertencer a determinada classe sendo que, quanto maior a área definida no treinamento, maior a precisão da classificação.

Após a classificação calculou-se as áreas de cada classe, bem como a matriz de confusão para verificar a precisão da classificação sem passar por nenhum tipo de correção. De acordo com Körting (2012, p. 43), as validações são necessárias pois "conhecer a precisão do mapa é vital para qualquer decisão tomada usando esse mapa".

A primeira avaliação foi a visual, combinação olho/cérebro humano. Isso porque os resultados, mesmo quando validados quantitativamente precisa convencer ao "olho humano" (BAATZ et al., 2000; GAMANYA et al., 2007, KÖRTING, 2012).

O procedimento de validação utilizando matriz de confusão, através do índice Kappa, para todas as classificações foi realizado no ArcGIS 10.6.1 levando em consideração pontos de referência de campo, tomados aleatoriamente, no momento dos trabalhos de campo (voo do VANT) e também pontos complementares (aleatórios) obtidos por fotointerpretação (visual) no ortomosaico.

\section{RESULTADOS E DISCUSSÃO}

A partir dos processos de classificação, a árvore de decisão foi representada graficamente, a fim de facilitar seu entendimento (Anexo 1). A mineração de dados considerou 37 atributos (espaciais e espectrais) para compor a árvore de decisão e atingir os resultados de cobertura da terra.

Os atributos e a porcentagem de importância para a decisão foram dados após o processamento das imagens no GeoDMA. Todavia, na tabela 1 são apresentados apenas os 15 atributos que obtiveram maior porcentagem de atuação no processo de classificação.

A quantidade de atributos considerados revela a complexidade da distinção de elementos (classes) diferenciáveis na imagem (Figura 3; Figura 4). Assim, corroborase com Martins (2012) quando o autor afirma que a extração de informação geográfica em grande escala em imagens de alta resolução é relevante em estudos de áreas urbanas.

Tabela 1. Atributos e bandas usados para a mineração de dados

\begin{tabular}{l|c}
\multicolumn{1}{c|}{ Atributo } & Uso do Atributo (\%) \\
\hline Razão da banda 2 & 100 \\
\hline Média da banda 3 & 45,42 \\
\hline Razão da banda 3 & 40,03 \\
\hline Razão da banda 1 & 33,15 \\
\hline Coeficiente da variação da banda 1 & 30,50 \\
\hline Desvio padrão da banda 3 & 25,49 \\
\hline Dissimilaridade da banda 3 & 22,67 \\
\hline Dissimilaridade da banda 1 & 20,53 \\
\hline Elipse circunscrita ao redor do objeto & 19,59 \\
\hline Razão entre perímetro do objeto e raiz quadrada da & 19,33 \\
área do objeto & 19,03 \\
\hline Energia da banda 3 & 16,77 \\
\hline Skewness da banda 1 & 14,76 \\
\hline Mediana da banda 3 & 14,71 \\
\hline Moda da banda 1 & \\
\hline
\end{tabular}

Fonte: Elaborado pelos autores (2019) 
A quantificação dos resultados a seguir inicia-se pela classificação da GEOBIA (mineração de dados), onde a classe de maior extensão mapeada foi o asfalto com $21,70 \%\left(4.142,38 \mathrm{~m}^{2}\right)$, seguido das árvores com $18,43 \%\left(3.518,32 \mathrm{~m}^{2}\right)$. No entanto, as classes de maior expressão do mapeamento são os telhados, que representam as áreas construídas. Os telhados de fibra apresentaram $12,54 \%\left(2.392,98 \mathrm{~m}^{2}\right)$, os telhados de cerâmica possuem $10,87 \%\left(2.074,16 \mathrm{~m}^{2}\right)$, seguidos dos telhados de metal com $3,75 \%\left(715,36 \mathrm{~m}^{2}\right)$. Juntas, as classes representam $5.182,49 \mathrm{~m}^{2}$. Foram mapeadas ainda as calçadas com $15,62 \%\left(2.982,53 \mathrm{~m}^{2}\right)$, terrenos com $4,72 \%$ $\left(901,22 \mathrm{~m}^{2}\right)$ e grama com $12,20 \%\left(2.329,85 \mathrm{~m}^{2}\right)$.

A característica mais marcante e de grande dificuldade na classificação de imagens de altíssima resolução são as diferenças na resposta espectral entre telhados. Embora seja uma mesma informação, a escala detalhada permite diferenciar muitos tipos de telhado (Figura 4).

Figura 3. Mapa de cobertura da terra pelo método de GEOBIA.

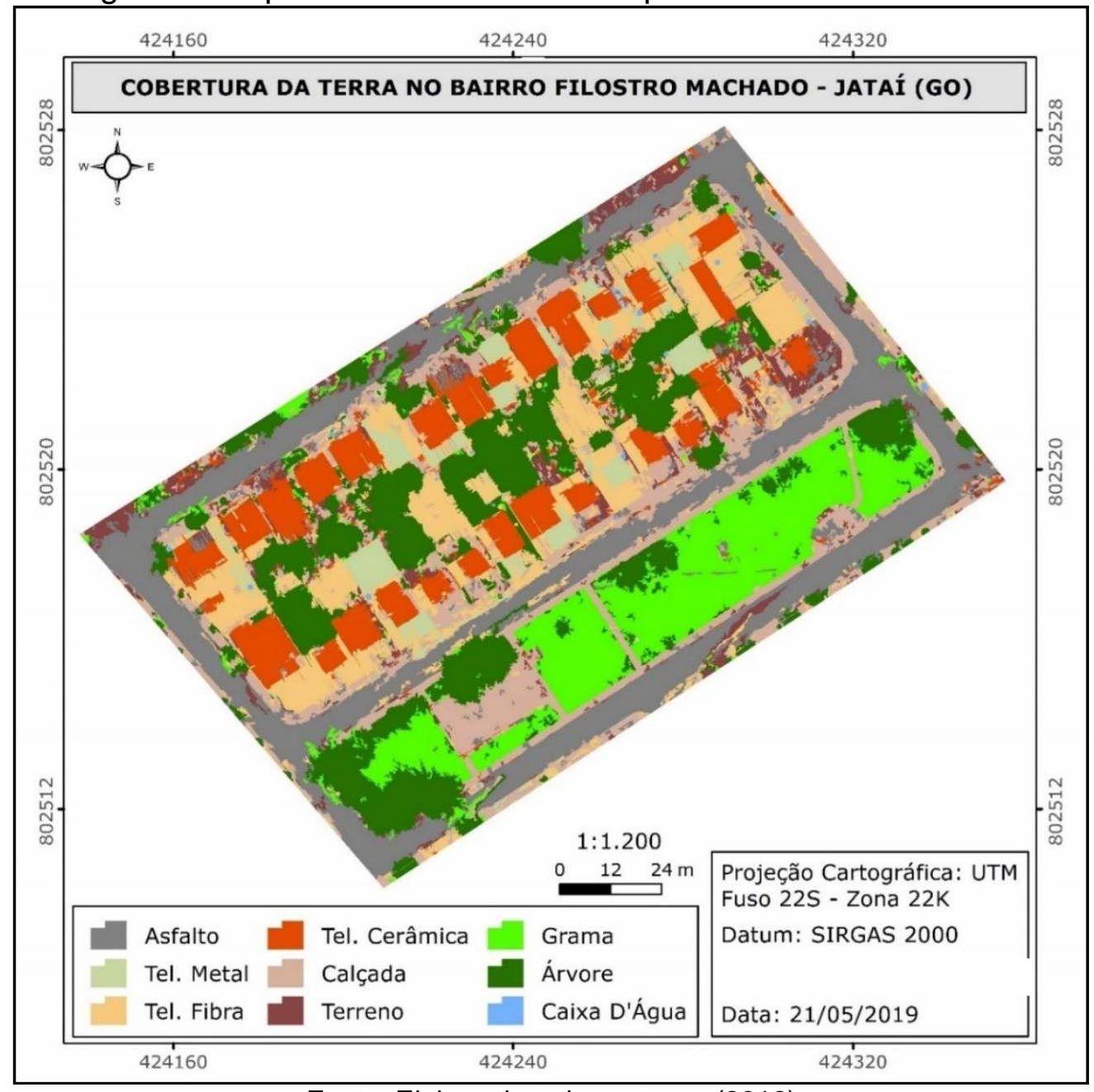

Fonte: Elaborado pelos autores (2019)

Mesmo numa área teste de extensão reduzida, definiu-se três tipos de telhados, de cerâmica, fibra e metal. Ainda assim, o nível de conservação e limpeza dos telhados interferem nas respectivas respostas espectrais (Figura 5). 
Figura 4. Gráfico de cobertura da terra pelo método de GEOBIA

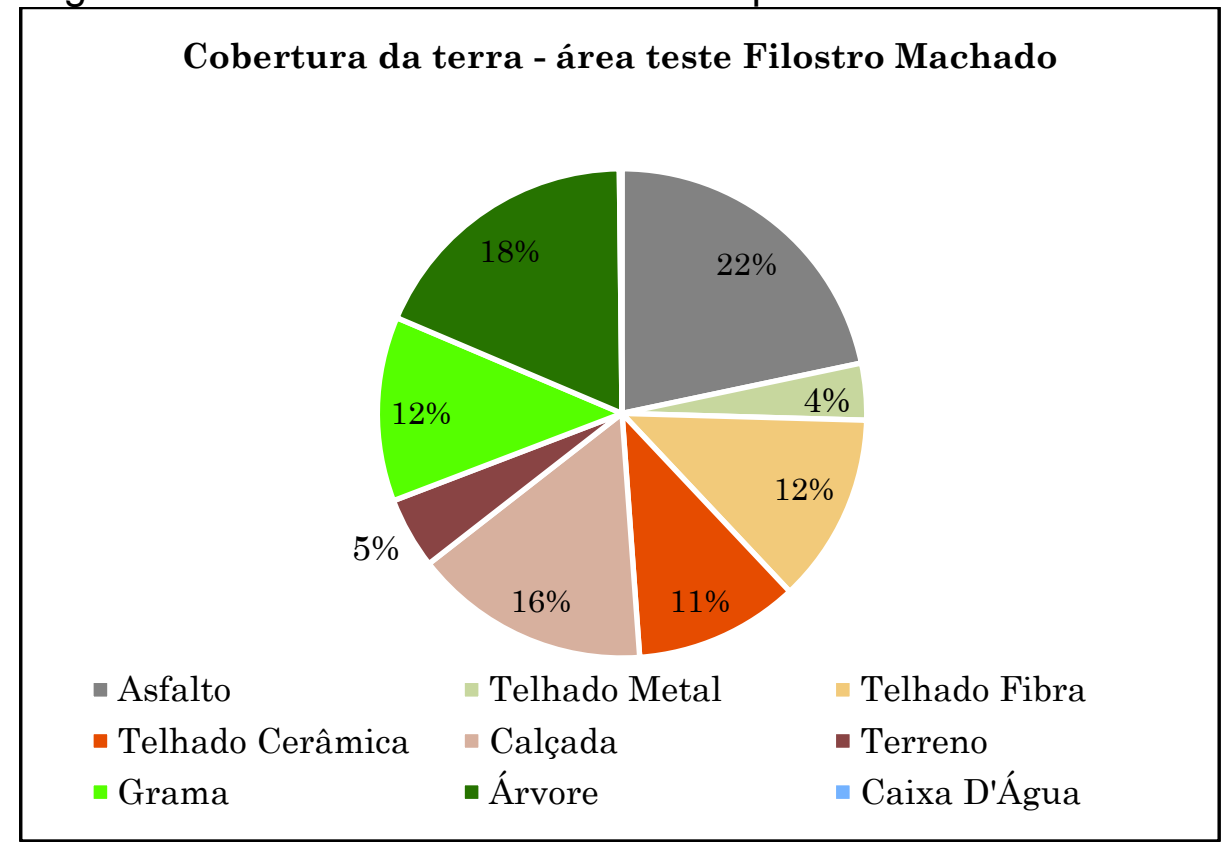

Fonte: Elaborado pelos autores (2019).

Figura 5. Diferentes respostas espectrais dos telhados mapeados: A) Telhado de cerâmica limpo; B) Telhado de metal limpo; C) Telhado de fibra limpo; D) Telhado de cerâmica sujo; E) Telhado de metal sujo; F) Telhado de fibra sujo

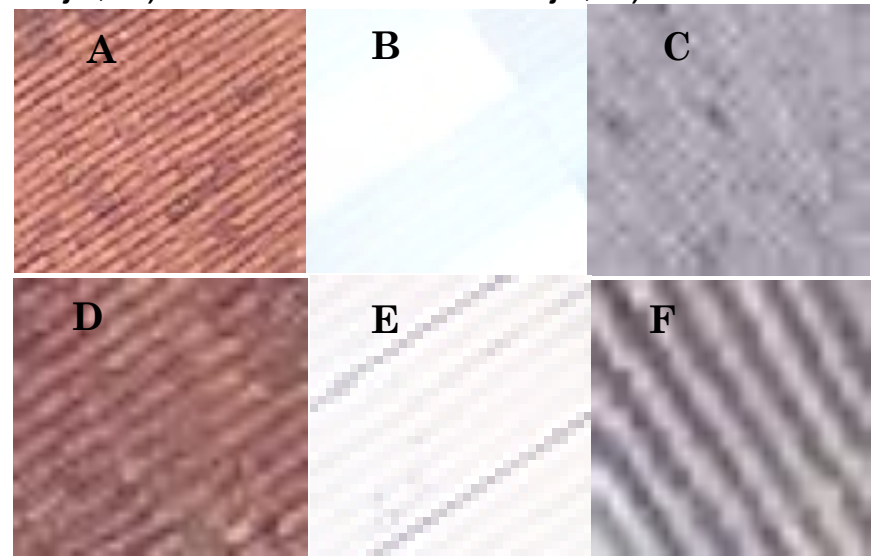

Fonte: Elaborado pelos autores (2019)

A priori, a classificação foi considerada satisfatória tendo vista o elevado número de classes e de alvos contidos na imagem do VANT. A primeira avaliação, visual, se mostrou razoavelmente satisfatória. A segunda avaliação, através da matriz Kappa, apresentou acerto de $82 \%$.

A porcentagem de erro calculada para o resultado da classificação foi compreendida como um valor esperado tendo em vista o alto nível de detalhamento em função da resolução espacial da imagem (Figura 6). 
Figura 6. Exemplo de confusão pela alta diferenciação espectral numa mesma classe

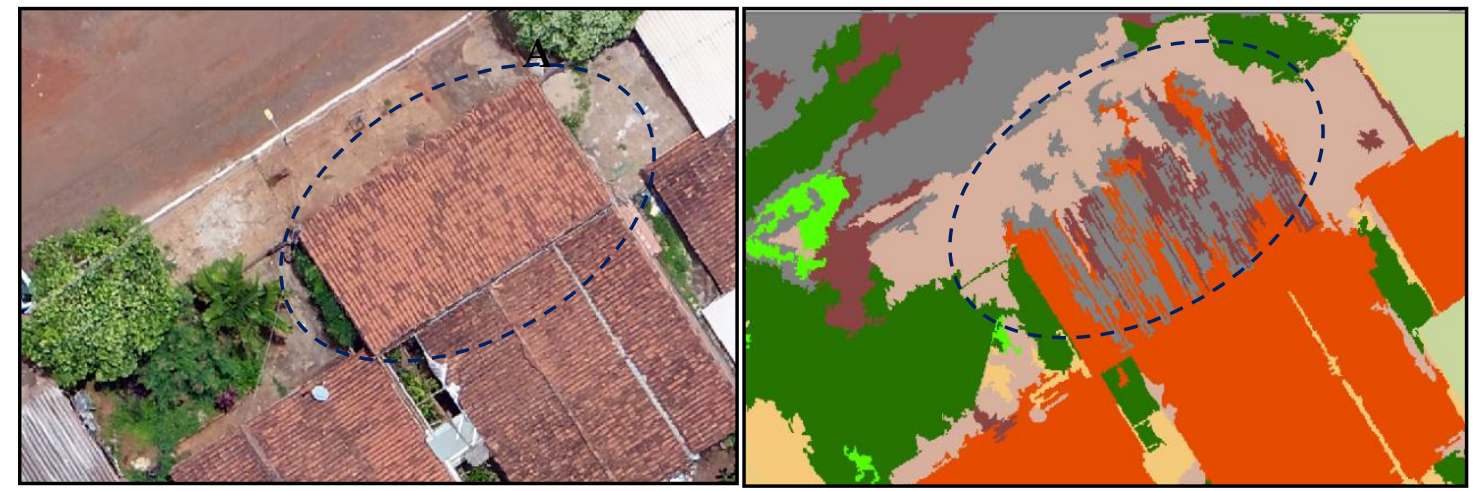

Fonte: Elaborado pelos autores (2019)

A comparação visual entre recortes da imagem e resultados da classificação permite verificar que extração de informações contextuais através da GEOBIA e a mineração de dados através dos atributos extraídos contribuíram para a diferenciação de muitas classes e para a qualidade da classificação, confirmando que essas são técnicas que rompem antigas barreiras marcadas por classificações baseadas estritamente em pixel ou segmentações apenas por valores espectrais.

Entre as maiores contribuições da GEOBIA e da mineração de dados é a possibilidade de não depender apenas da informação espectral das imagens.

Além da pura informação espectral, essa informação muitas vezes essencial, pode ser usada junto com as características de forma e textura dos objetos da imagem para melhorar significativamente a classificação [...]. Uma parte básica do procedimento é uma nova técnica para segmentação de objetos que é capaz de encontrar objetos de imagem em qualquer resolução escolhida (estruturas finas ou grosseiras) e alta qualidade (BAATZ e SCHÄPE, 1999, p. 1).

Comparativamente a outros classificadores mais comuns, a mineração de dados apresenta uma matriz com taxa de acerto maior (82\% de acerto) indicando concordância excelente apesar dos erros demonstrados. No processo de classificação supervisionada por Máxima Verossimilhança (Maximum Likelihood), a matriz de confusão indicou um percentual de acerto de $69 \%$, apontando para concordância substancial.

Já a classificação não supervisionada por clustering resulta em apenas $44 \%$ (concordância moderada), além de visualmente apresentar o efeito "sal e pimenta" evidenciando a grande confusão espectral quando o classificador se baseia estritamente no valor de cada pixel, sobretudo em imagens de VANT (Figura 7). 
Figura 7. Comparação entre a classificação: A) Não supervisionada - Iso Cluster; B) Supervisionada - Maximum Likelihood

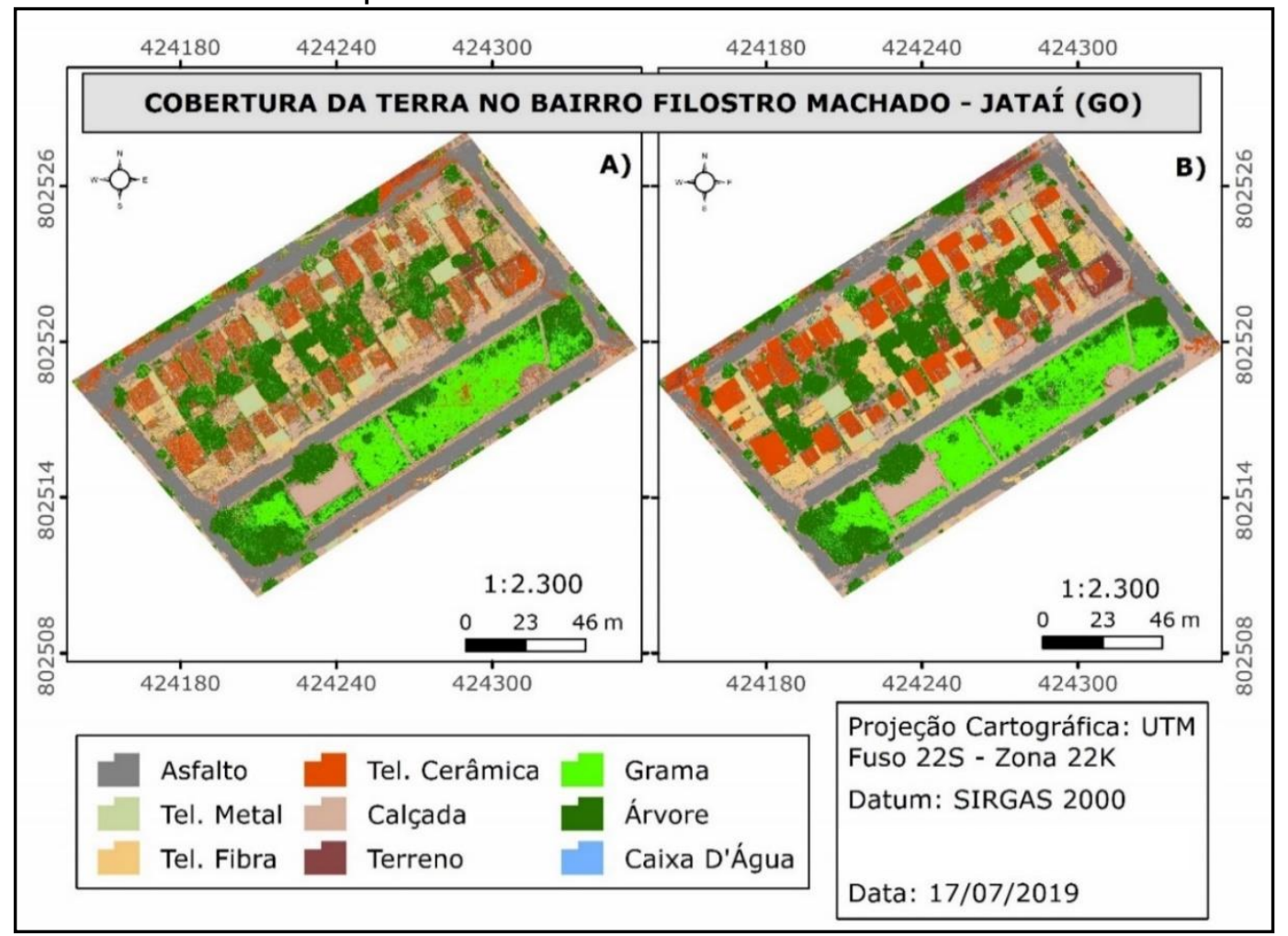

Fonte: Elaborado pelos autores (2019)

As maiores discrepâncias e, consequentemente, confusão nas matrizes ocorreram entre as classes telhado cerâmica, calçada, asfalto e terreno, visto a cor avermelhada dos solos da região que deixam estas categorias com tonalidades muito parecidas (tabela 2). Os métodos supervisionado e não supervisionado apresentaram ainda confusão entre as classes árvore e grama considerando a tonalidade da vegetação, aspecto suavizado na técnica de mineração de dados.

Tabela 2. Comparativo da quantificação das classes de cobertura da terra nas diferentes classificações

\begin{tabular}{lccc}
\hline $\begin{array}{l}\text { Categoria de } \\
\text { cobertura da } \\
\text { terra }\end{array}$ & $\begin{array}{c}\text { GEOBIA com } \\
\text { Mineração de } \\
\left.\text { dados } \mathbf{( m}^{2}\right)\end{array}$ & $\begin{array}{c}\text { Classificação } \\
\text { supervisionada por } \\
\text { máxima } \\
\text { verossimilhança }\left(\mathbf{m}^{2}\right)\end{array}$ & $\begin{array}{c}\text { Classificação } \\
\text { não- } \\
\text { supervisionada } \\
\text { por Iso Cluster } \\
\left(\mathbf{m}^{2}\right)\end{array}$ \\
\hline Árvore & $3.518,32$ & $3.963,00$ & $3.754,00$ \\
\hline Asfalto & $4.142,38$ & $4.093,00$ & $4.911,00$ \\
\hline Caixa d'água & 33,37 & 36,84 & 57,04 \\
\hline Calçada & $2.982,53$ & $3.071,00$ & $2.671,00$ \\
\hline Grama & $2.329,85$ & $2.157,00$ & $2.274,00$ \\
\hline $\begin{array}{l}\text { Telhado } \\
\text { cerâmica }\end{array}$ & $2.074,16$ & $2.302,00$ & $2.205,30$ \\
\hline Telhado fibra & $2.392,98$ & $2.152,00$ & $1.761,00$ \\
\hline Telhado metal & 715,36 & 671,30 & 789,30 \\
\hline Terreno & 901,22 & 643,80 & 667,70 \\
\hline
\end{tabular}


Os erros apresentados na classificação por GEOBIA apontam, primeiramente, para a necessidade de novos testes, sobretudo no que tange à escolha de atributos para a mineração de dados. Outro aspecto ligado aos resultados apresentados coloca em questão, além da qualidade da classificação de imagens de altíssima resolução, também a necessidade de classificação de imagens com tais características.

Além dos erros de classificação, um dos problemas enfrentados foi o da seleção da área teste. Tendo em vista que inicialmente a ortofoto cobria todo o setor Filostro Machado (Jataí - GO), contando ainda com uma maior diversidade de elementos. No entanto, por limitações de processamento do sistema de classificação, - GeoDMA, a ortofoto (área teste) precisou ser reduzida (recortada), considerando-se que o sistema possui limitações com relação ao volume de dados e não obteve êxito na extração de atributos da imagem que representava a área total do bairro.

Outra preocupação com relação à ampla utilização de VANTs para imageamento, são as dificuldades operacionais e legais apresentadas por tais plataformas. Conforme ressaltam Almeida et al. (2016), atualmente há uma enorme oferta de modelos no mercado, todavia poucos são bem adaptados e preparados para fins de mapeamentos.

Por vezes, os VANTs podem apresentar dificuldades para garantir sobreposições latitudinais e laterais, ocasionadas pela instabilidade da plataforma, a falta de exatidão na determinação doa parâmetros de posição e orientação das imagens através do receptor Global Navigations Satellite System (GNSS). Já as dificuldades legais se referem à indefinição de uma legislação nacional relativa à regulamentação de uso, procedimentos de segurança e voo e autorização para a mapeamentos, sobretudo em áreas urbanas (ALMEIDA et al., 2016).

Ainda assim, os resultados apresentados para classificação da imagem de VANT mostrou-se satisfatório e converge para, corroborando com Buffon, Paz e Sampaio (2017), auxiliar no nível de aproximação da realidade através da classificação imagens de altíssima resolução.

Ressalta-se, ainda, a necessidade de submeter estes resultados a processos de pós-classificação, preferencialmente manual, permitindo que o técnico-intérprete atinja um nível mais satisfatório de acerto e uma maior precisão na delimitação de classes.

\section{CONSIDERAÇÕES FINAIS}

A GEOBIA é considerada uma evolução no que tange à classificação de imagens em sensoriamento remoto. A mineração de dados representa, mais recentemente, um novo paradigma na para o processamento digital de imagens. Com os rápidos avanços de novos sensores orbitais e aerotransportados de altíssima resolução, além dos avanços referentes às resoluções espaciais e radiométricas, as próximas etapas exigirão cada vez mais possibilidades e inovações para obter bons resultados na extração da informação geográfica.

A classificação da cobertura da terra em áreas urbanas sempre representou um desafio, até mesmo em imagens de alta e média resolução espacial. Com a versatilidade no uso de VANTs para mapeamento urbano, se faz necessário avaliar o comportamento de técnicas disponíveis para seu uso. A GEOBIA junto da mineração de dados, apresentou resultados satisfatórios para uma classificação automática de imagens, sobretudo quando comparada a outros classificadores (por regiões e pixel a pixel). 
Todavia, alguns erros ainda indicam a necessidade de intervenção por meio do técnico-intérprete. Outra questão relevante a ser mencionada é da necessidade de classificação de imagens de altíssima resolução, diante de um cenário onde comumente opta-se pela vetorização (manual) das imagens devido à complexidade da classificação.

Em termos práticos, a classificação por GEOBIA e mineração de dados, embora tenha apresentado um resultado satisfatório, ainda exigiria atividades de pósclassificação para correção manual (vetorização) das confusões apresentadas. Com relação ao GeoDMA, em sua versão atual o software ainda não está totalmente preparado para essa estrutura de imagens, o que justifica o recorte e redução na áreateste, visto que o software apresenta limitações no input de dados raster, considerando sua estrutura (linhas e colunas).

Num futuro próximo, com a grande quantidade e disponibilidade de imagens de altíssima resolução espacial, o aprimoramento de técnicas para classificação e extração de informações automatizadas de imagens será essencial e a mineração de dados, traz agora essa perspectiva.

\section{REFERÊNCIAS BIBLIOGRÁFICAS}

ALMEIDA, I. C. et al. Estudo sobre o uso de Veículo Aéreo Não Tripulado (VANT) para mapeamento aéreo com fins de elaboração de projetos viários. Anais do Simpósio Brasileiro de Ciências Geodésicas e Tecnologias da Geoinformação, Pernambuco, 2016. pp. 1-10.

BAATZ, M.; SCHÄPE, A. Objected oriented and multiscale image analysis in semantic network. Proceedings of International Symposium on Operational Remote Sensing, Enschede, 1999. pp. 1-7

BAATZ, M.; SCHAPE, A.; SCHÄPE, M. Multiresolution segmentation: an optimization approach for high quality multi-scale image segmentation. Proceedings of Wichmann-Verlag, Heidelberg, 2000. pp. 12-23.

BLASCHKE, T. Object based image analysis for remote sensing. ISPRS Journal of Photogrammetry and Remote Sensing, vol. 65, p. 2-16, 2010.

BRAZ, A. M. et al. Análise da aplicação de VANT na atualização de cadastro florestal com uso de pontos de controle. Anais do Simpósio Brasileiro de Sensoriamento Remoto, João Pessoa, 2015. pp. 2751-2758.

BUFFON, E. A. M.; PAZ, O. L. S.; SAMPAIO, T. V. M. Uso de Veículo Aéreo Não Tripulado (VANT) para mapeamento das vulnerabilidades à inundação urbana: referenciais e bases de aplicação. Revista do Departamento de Geografia, vol. Especial - Eixo 9, p. 180-189, 2017.

CAMILO, C. O.; SILVA, J. C. Mineração de Dados: conceitos, tarefas, métodos e ferramentas. Relatório Técnico. Instituto de Informática (INF). Goiânia: UFG, 2009.

CHEN, Y., SU, W., Li, J., SUN, Z. Hierarchical object-oriented classification using very high resolution imagery and LIDAR data over urban areas. Advances in Space Research, vol. 43, p. 1101-1110, 2009. 
EHLERS, M.; JANOWSKY, R.; GAEHLER, M. New remote sensing concepts for environmental monitoring. Proceedings of Remote Sensing for Environmental Monitoring, GIS Applications, and Geology, Toulouse, 2002. pp. 1-12.

FAYYAD, U; SHAPIRO, G. P.; SMYTH, P. From data mining to knowledge discovery in databases. Al Magazine, Menlo Park, vol. 17, n. 3, p. 37-54, 1996a.

FAYYAD, U.; SHAPIRO, G. P.; SMYTH, P. The KDD process for extracting useful knowledge from volumes of data. Communications of the ACM, vol. 39, n. 11, p. 2734, nov., 1996b.

GAMANYA, R.; DEMAEYER, P.; DEDAPPER, M. An automated satellite image classification design using object-oriented segmentation algorithms: A move towards standardization. Expert Systems with Applications, v. 32, n. 2, p. 616-624, fev. 2007.

GeoDMA <2.0>. São José dos Campos, SP: Instituto Nacional de Pesquisas Espaciais (INPE), 2011. Disponível em <http://www.dpi.inpe.br/geodma>. Acessado em 08/03/2019.

GERKE, T. Mineração de dados de imagens obtidas com aeronave remotamente pilotada para estimativa de produtividade do trigo. Dissertação de mestrado. Universidade Estadual de Ponta Grossa, Programa de Pós-Graduação em Computação Aplicada, Ponta Grossa, 2017. 81p.

GOODCHILD, M. F. Spatial thinking and the GIS user interface. Procedia - Social and Behavioral Sciences, York, vol. 21, p. 3-9, 2011.

HAY, G. J.; CASTILLA, G. Geographic Object-Based Image Analysis (GEOBIA): a new name for a new discipline. In: BLASCHKE, T.; LANG, S.; HAY, G. J. (Org.). Objectbased image analysis: spatial concepts for knowledge-driven remote sensing applications. Berlin: Springer, 2008. p. 75-89.

KÖRTING, T. S. GeoDMA: a toolbox integrating data mining with object-based and multi-temporal analysis of satellite remotely sensed imagery. Tese de doutoramento. Instituto Nacional de Pesquisas Espaciais, Programa de Pós-Graduação em Sensoriamento Remoto, São José dos Campos, 2012. 96p.

KÖRTING, T. S.; FONSECA, L. M. G.; CAMARA, G. Interpreting Images with GeoDMA. Proceeding of Geographic Object-Based Image Analysis Conference, Ghente, 2010. pp. 1-5.

KÖRTING, T. S.; FONSECA, L. M. G.; CAMARA, G. GeoDMA - geographic data mining analyst a framework for GIScience. Computers \& Geosciences, Laramie, v. 57, p. 133-145, 2013.

LECHNER, A. M. Characterising upland swamps using object-based classification methods and hyper-spatial resolution imagery derived from an unmanned aerial 
vehicle. Proceedings of ISPRS Annals of the Photogrammetry, Remote Sensing and Spatial Information Sciences, Melbourne, 2012. pp. 101-106.

MARTINS, N. M. N. Utilização de imagens de satélite de alta resolução para a extracção de elementos em ambiente urbano. Dissertação de mestrado. Universidade de Lisboa, Mestrado em Engenharia Geográfica, Lisboa, 2012; 75p.

PILZ, H.; STROBL, J. Análise da impermeabilização com base em ortofotografias aéreas coloridas digitais através de processamento de imagens orientadas a objeto. In: BLASCHKE, T.; KUX, H. (Org.). Sensoriamento remoto e SIG avançados: novos sistemas sensores, métodos inovadores. Traduzido por Hermann Johann Heinrich Kux. 2. ed. São Paulo: Oficina de Textos, 2007. p. 162-171.

PUISSANT, A.; WEBER, C. The utility of very high spatial resolution images to identify urban objects, Geocarto International, Houston, vol. 17, n. 1, p.33-44, 2002. DOI: $10.1080 / 10106040208542223$

RUIZ, L. F. C.; GUASSELLI, L. A.; HACKMANN, C. L. Técnicas de mineração de dados empregadas na classificação de imagens obtidas por Veículo Aéreo Não Tripulado (Vant). Anais da Semana Geomática, Santa Maria, 2016. pp. 163-173. 